\title{
REMOCIÓN DE CROMO EN AGUAS RESIDUALES INDUSTRIALES MEDIANTE EL USO DE BIOMASA DE SPIRULINA SP, SEDIMENTACIÓN PRIMARIA Y PRECIPITACIÓN QUÍMICA
}

\author{
REMOVAL OF CHROMIUM IN INDUSTRIAL WASTEWATER \\ USING BIOMASS OF SPIRULINA SP, PRIMARY SEDIMENTATION \\ AND CHEMICAL PRECIPITATION
}

\begin{abstract}
Yeni Maritza Meneses Barroso ${ }^{1}$ Paula Andrea Patiño Mantilla ${ }^{2}$ Jhon Freddy Betancur ${ }^{3}$
\end{abstract}

\begin{abstract}
Est. Maestría en Desarrollo Sostenible y Medio Ambiente. Universidad de Manizales. Manizales, Colombia.

Est. Maestría en Desarrollo Sostenible y Medio Ambiente. Universidad de Manizales. Manizales, Colombia.
\end{abstract}

PhD Ciencias Agropecuarias, Universidad de Manizales; Grupo de Investigaciones Biomédicas, Centro de Investigación en Medio Ambiente y Desarrollo Sostenible - CIMAD (Manizales, Caldas, Colombia)

\footnotetext{
${ }^{1}$ yenime27@gmail.com

${ }^{2}$ paulaandreapatinomegmail.com

3 jbetancur@umanizales.edu.co
}

\section{RESUMEN}

El cromo es un metal pesado ampliamente usado a nivel industrial y vertido comúnmente de manera directa a fuentes hídricas, lo que conlleva a un deterioro de las mismas. Por lo tanto, esta investigación busca comprobar la remoción de cromo ejercida mediante tratamiento físico, químico y biológico en aguas residuales industriales, con el fin de determinar cuál alternativa resulta más sostenible.

Para ello se implementaron a escala laboratorio ocho reactores con agua residual industrial de una empresa del sector metalmecánico; inicialmente se evaluó la remoción de cromo mediante sedimentación primaria logrando remociones del 16\%; posteriormente, se verificó el efecto llevado a cabo mediante precipitación química con sulfato de aluminio y oxido de calcio; por último, se evaluó la capacidad 
de biosorción de la microalga Spirulina sp utilizando biomasa viva y muerta a las 24 y 96 horas. Encontrando que existen mecanismos de fácil implementación para evitar vertimientos directos, además se encontró que el tratamiento químico logra disminuir el cromo a valores inferiores de $0.1 \mathrm{mg} / \mathrm{l}$, con remociones del $99 \%$, sin embargo, genera más del $30 \%$ de lodos y no favorece la separación del metal. Con el uso de biomasa se alcanzaron remociones de hasta el $96.5 \%$ en las primeras 24 horas y un valor final de cromo de 1.02 $\mathrm{mg} / \mathrm{l}$, presentando la ventaja que este método genera menos del $10 \%$ de lodos y permite recuperar el cromo para su posterior uso. De modo que, se recomienda la implementación de un tratamiento físico-biológico, puesto que mejora la sostenibilidad del proceso.

Palabras clave: agua residual industrial, biosorción, cromo, microalga, Spirulina sp.

\section{ABSTRACT}

Chromium is a heavy metal widely used at an industrial level and commonly discharged directly into water sources, which leads to their deterioration. Therefore, this research seeks to verify the removal of chromium exerted by physical, chemical and biological treatment in industrial wastewater, in order to determine which alternative is more sustainable.
With this aim, three reactors with industrial wastewater from a company in the metal-mechanic sector were tested; Chromium removal was initially evaluated by primary sedimentation achieving $16 \%$ removals; subsequently, the effect carried out by chemical precipitation with aluminum sulfate and calcium oxide was verified. Finally, the bio-absorption capacity of the microalga Spirulina $s p$ was evaluated using living and dead biomass within 24 and 96 hours. Results show that there are mechanisms with easy implementation to avoid direct dumping. It was also found that the chemical treatment reduces chromium to values lower than $0.1 \mathrm{mg} / \mathrm{l}$, with removals of $99 \%$, however, it generates more than $30 \%$ of sludge and does not help to separate the metal. With the use of biomass, removals of up to $96.5 \%$ were achieved in the first 24 hours and a final chromium value of $1.02 \mathrm{mg} / \mathrm{l}$, with the advantage that this method generates less than $10 \%$ of sludge and allows the recovery of chromium for its later use. Therefore, the implementation of a physical-biological treatment is recommended, since it allows improving the sustainability of the process.

Keywords: industrial wastewater, biosorption, chromium, microalgae, Spirulina sp. 
TRATAMIENTO FÍSICO

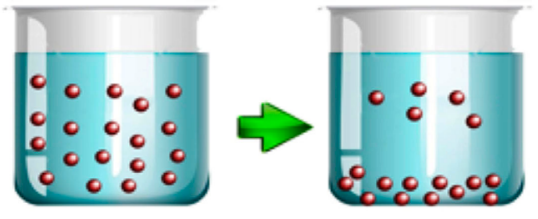

Remoción: $16 \%$

Residuos $<5 \%$

Recuperable

TRATAMIENTO FÍSICO - QUÍMICO

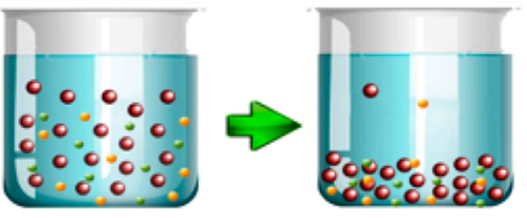

Remoción: $99.6 \%$

Residuos > 30\%

No Recuperable

TRATAMIENTO FÍSICO - BIOLÓGICO
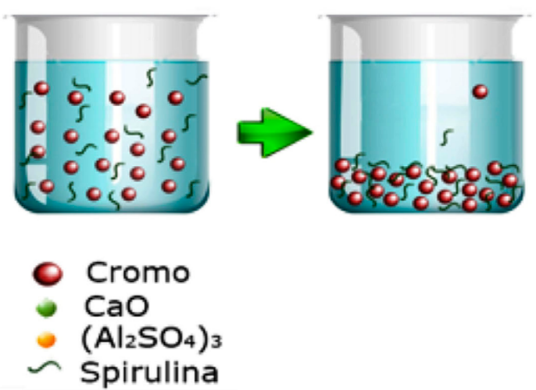

Remoción: $96.5 \%$

Residuos: < 10\%

Recuperable

Figura 1: Resultados con los diferentes tipos de tratamiento propuestos. Porcentaje de remoción de cromo, generación de residuos y recuperabilidad del cromo.

\section{INTRODUCCIÓN}

A través del tiempo la actividad antrópica se ha encargado de alterar de una forma acelerada el entorno natural. Es así como los seres humanos han afectado la calidad de las fuentes hídricas, no sólo a través de los vertimientos domésticos, sino también desde los diferentes procesos industriales que se desarrollan para soportar la economía. Dentro de ellos está el sector industrial que en muchos casos vierte sus residuos líquidos de manera directa a los cuerpos de agua sin los tratamientos adecuados. Es así como ciertas industrias utilizan a manera de insumo en sus procesos productivos metales pesados como el cromo, el plomo, el mercurio, el cadmio y el zinc entre otros; dichos compuestos son considerados entre los más peligrosos para la salud humana y el medio natural, por lo que resulta fundamental evitar su vertimiento a las fuentes hídricas.

En depuración de aguas residuales industriales, en general, se realizan diversos procesos para llevar el agua a parámetros admisibles por la normatividad ambiental, sin embargo, la eliminación de los metales pesados resulta compleja y dispendiosa, además presenta el inconveniente que todos los tipos de tratamientos generan a su vez residuos (López, González, \& Guzmán, 2016). Estos autores explican que el tratamiento de aguas residuales se divide en cuatro etapas que son: pretratamiento (eliminación de sólidos gruesos 
en suspensión, grasas y aceites), tratamiento primario (remoción de una porción de sólidos suspendidos y materia orgánica mediante la gravedad), tratamiento secundario (remoción de sólidos suspendidos y materia orgánica) y tratamiento terciario (remoción de sustancias residuales no eliminadas por los procesos secundarios entre ellas el cromo (Cr)). El propósito de esta investigación se centra en el cromo debido al potencial mutagénico, genotóxico, carcinogénico y teratogénico que genera en la salud de humanos y animales, dependiendo de la dosis expuesta, según lo reporta la Organización Mundial de la Salud (OMS) (2006) en la evaluación de riesgos del cromo.

El cromo existe en nueve estados de valencia, así: $-2,-1,0,+1,+2,+3,+4,+5,+6$, pero se manifiesta en el medio ambiente más comúnmente como compuestos de $\mathrm{Cr}$ (III), $\mathrm{Cr}$ (VI) y en la forma metálica $\mathrm{Cr}(0)$. De acuerdo con Park et al. (2005), citado por Martínez et al., (2015, pág. 14) los métodos más empleados en la eliminación de iones $\mathrm{Cr}(\mathrm{VI})$ implican la reducción química a iones de $\mathrm{Cr}$ (III), el cual es menos tóxico y menos soluble y la precipitación química como $\mathrm{Cr}(\mathrm{OH})_{3}$ utilizando una solución alcalina. Así mismo, existen otros métodos convencionales tales como adsorción con carbón activado, electrocoagulación, ultrafiltración, intercambio iónico con resinas, ósmosis inversa, filtración con membranas y fotocatálisis (Caviedes et al., 2015). Sin embargo, la eficiencia de estos métodos disminuye cuando la concentración de cromo en el agua es baja, entre ( 1 - $100 \mathrm{mg} / \mathrm{l})$, por lo que su aplicación es rentable para altas concentraciones de $\mathrm{Cr}$ generadas en grandes empresas que cuentan con los recursos suficientes para implementarlos en sus plantas de tratamiento de aguas residuales (Ardila, 2012).

Debido a que diversos autores sugieren que los tratamientos biológicos son una manera adecuada de tratar los sedimentos generados durante las fases del tratamiento de aguas. Peña, Cuesta, \& Perez (2015) indican que, para cumplir con las normativas actuales se deben crear nuevas alternativas que permitan la remoción de los contaminantes, entre ellas propone la biorremediación, degradación enzimática, la remediación microbiana y la fitorremediación, esta última también sugerida por Franco et al., (2016) quienes destacan que los sistemas biológicos contribuyen a mejorar los índices de calidad de agua y su biodegradabilidad. Así mismo, se resalta el potencial que tienen las microalgas en el tratamiento de aguas residuales (Duque, 2017).

Otro autores como Perez \& Labbe (2014) destacan que el uso microalgal sirve para la reducción de compuestos contaminantes y mencionan la importancia de su uso en la producción de biocombustibles. Así mismo, Duque (2017) resalta el uso de microalgas como una fuente potencial de energía de manejo asequible y más productiva dentro de las alternativas existentes de biocombustibles. Igualmente, Hernández et al. (2009) evaluaron el uso microalgal como materia prima en la obtención de biocombustibles y enfatizaron en la posibilidad de reutilizar los residuos microalgales después de la extracción de lípidos y de este modo hacer uso de los residuos del proceso y dar un valor agregado. Es así como, Martínez, Paredes, \& Ordoñes (2016) hacen énfasis en el uso que se le puede dar a la biomasa seca y explican cómo diversos tipos de biomasa son de gran utilidad, gracias a la capacidad interporal existente en ella; además resaltan el efecto bio-adsorbente que generan las microalgas en las aguas residuales industriales.

Cabe mencionar que las microalgas se encuentran presentes en todos los cuerpos de agua, incluso en el suelo y en la mayoría de los ambientes terrestres, adaptadas a condiciones 
extremas como son pH muy ácidos o muy alcalinos, altas y bajas temperaturas y alto contenido de sales y de metales (Castañeda \& Castillo, 2016). Estas condiciones son comunes al interior de las industrias, por lo que se requieren alternativas de tratamientos eficientes ajustados a estas características, que conlleven a evitar pasivos ambientales. Así mismo, el uso de microalgas se destaca por su capacidad depurativa, el poco espacio que requieren y el potencial reductor de metales pesados, tal como lo plantea Jbari (2012), quien además menciona los adelantos que se han realizado para remediar y descontaminar cuerpos de agua. Sin embargo, el uso de estos microorganismos en la remoción de metales requiere mayor investigación, por lo que se planteó el uso de biomasa viva y muerta de Spirulina $s p$ para la remoción de cromo presente en aguas residuales industriales, teniendo en cuenta su capacidad reductora de metales, tamaño celular $(10-500 \mu \mathrm{m})$, adaptabilidad a ambientes extremos y fácil cultivo.

Por lo anterior, se propuso en esta investigación verificar y comparar la remoción de cromo de aguas residuales generadas en una empresa metalmecánica como unidad de estudio, a partir de cuatro alternativas de tratamientos como son: tratamiento primario de sedimentación, coagulación-precipitación química, uso de biomasa viva y biomasa muerta de la microalga Spirulina sp.

\section{MATERIALES Y MÉTODOS}

Área de estudio. Teniendo en cuenta información suministrada por la Autoridad Ambiental Regional-Corpocaldas del departamento de Caldas (Colombia) sobre las caracterizaciones de agua residual a nivel empresarial se encontró que, para la ciudad de Manizales, el sector que mayores aportes genera es el sector metalmecánico. Con base en esto se realizó un listado de las empresas relacionadas con contaminación con cromo, con el fin de realizar el muestreo puntual en cada empresa y el respectivo análisis de cromo total. De acuerdo con los resultados obtenidos se seleccionó una empresa del sector metalmecánico con un valor de cromo total de $32,56 \mathrm{mg} / \mathrm{l}$ en sus aguas residuales. Los ensayos se realizaron a temperatura ambiente en un intervalo entre $25-32^{\circ} \mathrm{C}$ y una temperatura promedio de $26,5^{\circ} \mathrm{C}$.

Para desarrollar la fase experimental se utilizó agua residual industrial, agua residual industrial tratada físicamente por sedimentación, agua residual tratada por coagulación- precipitación química por adición de sulfato de aluminio y cal y una solución sintética contaminada con dicromato de potasio denominada solución patrón como punto de referencia. Los ensayos con biomasa viva y muerta de Spirulina $s p$ se realizaron en agua residual, agua residual sedimentada y en la solución patrón, esto con el propósito de verificar la eficiencia de remoción de cromo con los diferentes tipos de biomasa y finalmente determinar cuál alternativa entre el uso de biomasa de Spirulina $s p$, tratamiento físico por sedimentación primaria y precipitación química resulta ser más sostenible para remover cromo de aguas residuales industriales.

\section{Remoción de cromo de aguas residuales industriales mediante tratamiento físico} y precipitación química. Para determinar la eficiencia de remoción de cromo mediante tratamiento primario de tipo físico y químico, se tomó agua residual industrial (ARI) y fue sometida a los siguientes tipos de tratamiento:

- ARIS: Agua residual industrial sometida a tratamiento físico de sedimentación primaria.

- ARIT: Agua residual industrial sedimentada con posterior tratamiento químico con adición de cal $(\mathrm{CaO})$ a razón de 2,6 g/l para neutralizar el $\mathrm{pH}$ entre (6-7), debido a que el 
agua residual industrial presentó un $\mathrm{pH}$ inicial entre (1-2), el cual fue medido mediante método potenciométrico. Posteriormente, se utilizó sulfato de aluminio $\left(\mathrm{Al}_{2} \mathrm{SO}_{4}\right)_{3}$ como agente coagulante. Para determinar la dosis óptima de sulfato de aluminio se realizó una prueba de jarras a diferentes dosis del coagulante, así: $100 \mathrm{mg} / \mathrm{l}, 80 \mathrm{mg} / \mathrm{l}, 60 \mathrm{mg} / \mathrm{l}, 40 \mathrm{mg} / \mathrm{l}, 20$ $\mathrm{mg} / \mathrm{l}$, de las cuales se evidenció una mejor formación de floc a una dosis de $20 \mathrm{mg} / \mathrm{l}$.

A cada muestra de agua se le analizó Cr total mediante Digestión-Absorción Atómica- Llama directa aire acetileno (SM 3030 E) (SM 3111 B). El diseño del ensayo se puede apreciar en la Figura 2.

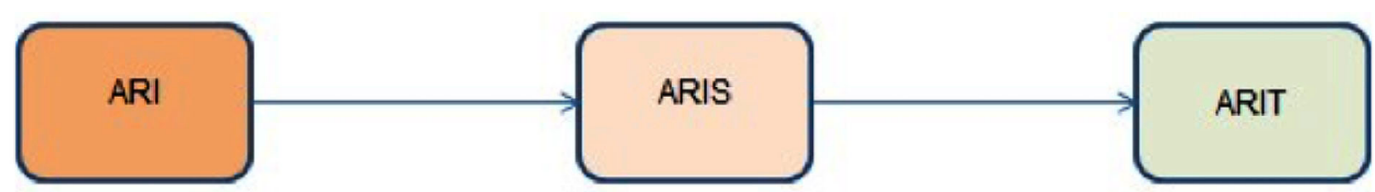

Figura 2. Diseño del ensayo realizado para el Tratamiento Físico y Químico.

El Porcentaje de remoción de cromo total fue calculado mediante la fórmula de eficiencia de remoción, relacionada en la ecuación 1.

Concentración inicial-Concentración final

Concentración inicial

$X 100$

(Ecuación 1)

Determinación de la eficiencia de remoción de cromo a partir de biomasa viva y biomasa muerta de Spirulina sp. El diseño experimental consistió en el uso de ARI (Agua residual industrial), ARIS (Agua residual sedimentada) y Solución patrón (compuesta por $\mathrm{K}_{2} \mathrm{Cr}_{2} \mathrm{O}_{7}$ y enriquecida con medio de cultivo $\mathrm{Za}$ rrouk) a una concentración de $41 \mathrm{mg} / \mathrm{l}$, debido a que se buscó trabajar a una concentración de cromo total igual o por encima de la encontrada en la unidad de estudio y de este modo tener un referente de remoción de cromo. Cada tipo de agua fue tratada con biomasa viva y muerta como se plantea en la Figura 3.

Para determinar la capacidad de remoción de cromo a partir de biomasa viva se añadió a cada reactor cepa de Spirulina $s p$ a una concentración de $1.55 * 10^{5}$ células $/ \mathrm{ml}$ correspondiente a $20 \mathrm{~g} / \mathrm{l}$. En cada reactor se verificó remoción a las 24 y 96 horas, teniendo en cuenta la dosis de biomasa y tiempos de contacto utilizados por Hong \& Shan-shan (2005) para la remoción de plomo mediante biomasa viva de Spirulina $s p$.

Los ensayos se realizaron a escala laboratorio en seis bioreactores tipo batch con un volumen de reacción de 2 litros, inyección de aire mediante un compresor de capacidad de 2,9 $W$ y medición de temperatura. Con el fin de analizar cromo en los diferentes tiempos, se tomaron alícuotas de $500 \mathrm{ml}$ y se filtraron con una tela de 40 micras de porosidad (liencillo) y embudo con el fin de tamizar la microalga para los respectivos análisis del agua, los cuales fueron realizados mediante el método de Digestión-Absorción Atómica- Llama directa aire acetileno (SM 3030 E) (SM 3111 B).

Para determinar la capacidad de remoción con biomasa muerta se utilizó una dosis de $6 \mathrm{~g} / \mathrm{l}$ de Spirulina sp verificando remoción a las 24 y 96 horas, teniendo en cuenta la dosis de biomasa y tiempos de contacto utilizados por García 
Hernández (2014) para la remoción de cromo mediante biomasa muerta de Spirulina sp. Estos ensayos se realizaron en reactores tipo batch con capacidad de 2 litros con agitación inicial para homogenizar la solución, dicha agitación se realizó antes de tomar cada muestra.
Para realizar el muestreo de este experimento se tomaron alícuotas de $500 \mathrm{ml}$ y se filtraron con el liencillo y embudo mencionados anteriormente, con el fin de tamizar la microalga para los respetivos análisis del agua a las $24 \mathrm{~h}$ y $96 \mathrm{~h}$ respectivamente (Ver Figura 3).
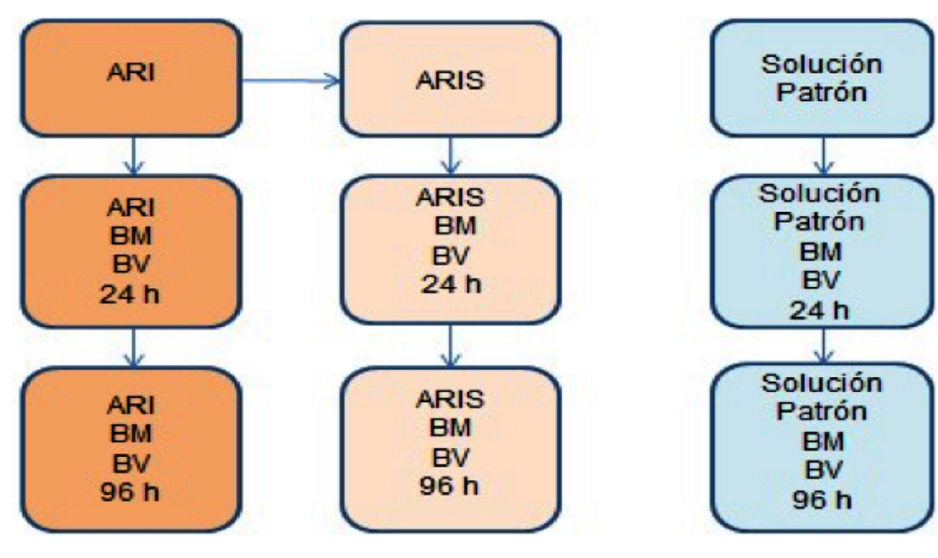

Figura 3. Diseño del ensayo realizado con biomasa viva (BV) y biomasa muerta (BM).

\section{Resultados y Discusión}

Eficiencia de remoción de cromo de aguas residuales industriales mediante tratamiento físico - químico.

En la Tabla 1 se puede apreciar los resultados obtenidos de cromo total en cada una de las alícuotas tomadas y las respectivas remociones llevadas a cabo en cada tipo de tratamiento. El agua residual fue sometida a tratamiento físico de sedimentación primaria encontrándose una remoción de cromo del $16 \%$ y una generación de aproximadamente el $5 \%$ de lodos.
Así mismo, esta fue sometida a un proceso de coagulación-precipitación química obteniendo una remoción de cromo total del 99,6\%. Cabe mencionar que se analizó $\mathrm{Cr}(\mathrm{VI})$ en cada reactor presentando un resultado de $<0.04 \mathrm{mg} / \mathrm{l}$, el cual es el límite de cuantificación del método utilizado para medir cromo hexavalente; Colorimetría (SM 3500-Cr-B). Por lo tanto, se infiere que el cromo total presente en el agua residual se encuentra como $\mathrm{Cr}$ (III).

Tabla 1. Resultados y eficiencia de remoción de cromo con tratamiento físico-químicos.

\begin{tabular}{|c|c|c|c|}
\hline Tratamientos & $\begin{array}{l}\text { Cromo total inicial } \\
(\mathrm{mg} / \mathrm{I})\end{array}$ & $\begin{array}{l}\text { Cromo total } \\
\text { Final }(\mathrm{mg} / \mathrm{I})\end{array}$ & Porcentaje de remoción \\
\hline ARI-ARIS & 29,3 & 24,7 & $16 \%$ \\
\hline ARIS-ARIT & 24,7 & 0,1 & $99,60 \%$ \\
\hline
\end{tabular}


De acuerdo con los resultados obtenidos, se pudo concluir que realizar tratamiento físico mediante sedimentación es ineficiente ya que en este estudio se demostró una remoción de cromo del 16\%. Sin embargo, el uso de un tratamiento de coagulación química con sulfato de aluminio y precipitación básica con cal puede generar remociones superiores al $99 \%$ para cromo total, lo que resulta efectivo para lograr valores inferiores a 0.5 $\mathrm{mg} / \mathrm{l}$, que coincide con el límite establecido en la Resolución 0631 de 2015 del Ministerio de Ambiente y Desarrollo Sostenible (MADS) (2015) para el caso de actividades de tratamiento y revestimiento de metales. Sin embargo, aunque resulta efectivo se debe tener en cuenta que aproximadamente el $30 \%$ del volumen del agua tratada se convierte en lodos, los cuales deben ser tratados y dispuestos de manera secundaria. Ortiz \& Carmona
(2015) y Tovar, Ortiz \& Jaraba (2015) coinciden en indicar que la formación, disposición y almacenamientos de lodos y desechos, es de difícil manejo y no es posible hacer recuperación del cromo. Por lo tanto, con el tratamiento químico no es factible separar y recuperar el metal de los sedimentos para su posterior aprovechamiento.

\section{Determinación de la eficiencia de remo- ción de cromo a partir de biomasa viva y biomasa muerta de Spirulina sp.}

Los análisis de cromo total con biomasa viva y muerta de Spirulina $s p$ se realizaron a las 24 y 96 horas tal como se observa en la Tabla 2. Los experimentos se realizaron en agua residual industrial (ARI), agua residual industrial sedimentada (ARIS) y en solución sintética denominada solución patrón, la cual contuvo como contaminante dicromato de potasio.

Tabla 2. Resultados y eficiencia de remoción de cromo con biomasa viva y muerta

\begin{tabular}{|c|c|c|c|}
\hline Ensayos & $\begin{array}{l}\text { Cromo total inicial } \\
(\mathrm{mg} / \mathrm{I})\end{array}$ & $\begin{array}{l}\text { Cromo total } \\
\text { Final }(\mathrm{mg} / \mathrm{I})\end{array}$ & $\begin{array}{l}\text { Porcentaje } \\
\text { de remoción }\end{array}$ \\
\hline \multicolumn{4}{|c|}{ Biomasa Viva } \\
\hline ARI 24 h & 29,3 & 1,02 & $96,50 \%$ \\
\hline ARI $96 \mathrm{~h}$ & 29,3 & 14,2 & $48,48 \%$ \\
\hline ARIS 24 h & 24,7 & 21,7 & $12 \%$ \\
\hline ARIS $96 \mathrm{~h}$ & 24,7 & 22,7 & $8 \%$ \\
\hline Solución patrón 24 h & 41 & 0,8 & $98 \%$ \\
\hline Solución patrón 96 h & 41 & 40,2 & $2 \%$ \\
\hline \multicolumn{4}{|c|}{ Biomasa Muerta } \\
\hline ARI 24 h & 29,3 & 20,3 & $30,70 \%$ \\
\hline ARI $96 \mathrm{~h}$ & 29,3 & 22,6 & $22,80 \%$ \\
\hline ARIS 24 h & 24,7 & 21,2 & $14 \%$ \\
\hline ARIS 96 h & 24,7 & 23,9 & $3 \%$ \\
\hline Solución patrón 24 h & 41 & 24,8 & $39,51 \%$ \\
\hline Solución patrón 96 h & 41 & 40,1 & $2 \%$ \\
\hline
\end{tabular}


En los análisis realizados con biomasa viva se encontró que en el agua residual industrial se lograron remociones de $\mathrm{Cr}$ total del 96,50\% a las 24 horas y $48,48 \%$ a las 96 horas. Para agua residual industrial sedimentada el porcentaje de remoción fue del $12 \%$ y $8 \%$, respectivamente y para la solución patrón fue de $98 \%$ y $2 \%$ en cada periodo de tiempo. En general, se evidencia una eficiencia de remoción superior a las 24 horas, ya que a las 96 horas la biosorción se revierte. Esto indica que hay desorción después de las 24 horas, ya que la microalga retiene lo que necesita para su metabolismo y expulsa el excedente.

En cuanto a las eficiencias encontradas utilizando biomasa muerta, después de 24 y 96 horas de tratamiento de agua residual industrial se evidencio $30,7 \%$ y $22,8 \%$ de remoción, respectivamente. Para el agua residual industrial sedimentada la remoción fue del $14 \%$ y $3 \%$ respectivamente. Para la solución patrón fue de $39,51 \%$ y $2 \%$ en cada periodo de tiempo. Al igual que con la biomasa viva, se generó una desorción o liberación del contaminante posterior a las 24 horas, debido al proceso de degradación de la biomasa muerta. Cabe mencionar que tras 48 horas, el agua generó fuertes olores propios de la materia orgánica en estado de descomposición.

Los resultados obtenidos permiten comprender que el cromo sigue estando presente en la solución. Es por esto que se puede inferir que el proceso que se está generando está asociado a la fisisorción, donde la partícula no se fija en un lugar específico, sino que está libre para trasladarse en la solución, conservando su naturaleza química. (Tovar, Ortiz, \& Jaraba, 2015).

La relación entre la remoción de cromo con biomasa viva y biomasa muerta está dada por dos situaciones; el tamaño y la capacidad poral. En primer lugar, la remoción con biomasa viva fue mayor debido al tamaño de la microalga, pues ésta es una microalga que tiene un tamaño entre 10 a $500 \mu \mathrm{m}$, por lo tanto, presenta mayor área superficial donde se pueden localizar los residuos o partículas del contaminante. Se infiere además que, el proceso mencionado por Tovar, Ortiz \& Jaraba (2015) de fisisorción fue el presentado en esta investigación, teniendo en cuenta la agitación del agua al momento de la toma de cada alícuota, por efecto hidrodinámico de las partículas que están en la superficie de la microalga, estas son liberadas nuevamente al medio. Teniendo en cuenta los resultados de la tabla 2 para biomasa viva, se infiere que el cromo continúa presente en el medio y en consecuencia se generó una desorción del contaminante.

En segundo lugar, la biomasa muerta pierde dos terceras partes de su tamaño y aunque no tiene tanta área superficial como la biomasa viva, por ser seca tiene la capacidad de rellenar las áreas intersticiales porales con los contaminantes a modo de adsorbente, además la degradación generada en la biomasa muerta es mucho más rápida debido a los procesos de desintegración. Por lo tanto, se da una liberación más rápida de lo almacenado, como se evidenció en los resultados obtenidos para biomasa muerta, tal como se muestra en la tabla 2.

Otro factor a tener en cuenta con la biomasa viva y muerta es el tiempo de degradación de cada una de estas, debido a que la biomasa seca ha perdido humedad y tamaño, lo que genera que se aumente su capacidad poral. Entre tanto la biomasa viva mantiene tamaño y debe deshidratarse para iniciar su proceso de descomposición lo que conlleva un mayor tiempo de degradación, como se puede verificar al comparar las remociones después de 96 horas con biomasa viva y biomasa seca.

Hong \& Shan-shan (2005) estudiaron el potencial que tiene la Spirulina $s p$ en estado vivo para 
la biorremediación de metales pesados como el plomo encontrando una biosorción del metal del $95 \%$ dentro de las $24 \mathrm{~h}$. Los resultados experimentales reportados en esta investigación son similares a las $24 \mathrm{~h}$ logrando una eficiencia de remoción del $96,5 \%$ y del $98 \%$ para agua residual industrial y agua sintética, respectivamente.

García Hernández (2014) estudió la remoción de cromo de agua sintética utilizando biomasa de Spirulina máxima, encontrando una eficiencia de remoción de $23,67 \%$ de $\operatorname{Cr}$ (III) en las primeras 72 horas para una concentración inicial de $101.72 \mathrm{mg} / \mathrm{l}$ y dosis de biomasa de $2 \mathrm{~g} / \mathrm{l}$. Resultados análogos con esta investigación, teniendo en cuenta las primeras $24 \mathrm{~h}$ de contacto biomasa-solución patrón, ya que en este estudio fue utilizada una dosis tres veces mayor de biomasa, lo que requiere menor tiempo de contacto.

\section{CONCLUSIONES}

La eficiencia de remoción de cromo mediante un proceso físico de sedimentación fue del $16 \%$, lo que resulta importante, ya que es una alternativa económica de fácil implementación en las pequeñas y medianas empresas y permite contribuir a mejorar la calidad del agua antes de la implementación de un tratamiento químico y/o biológico.

La remoción de cromo a partir de adición de cal para mantener un pH óptimo de coagulación con sulfato de aluminio, generó una remoción de cromo total de más de $99 \%$. Sin embargo, este tipo de tratamiento a su vez implica la generación de grandes volúmenes de lodos activos (aproximadamente el 30\% en volumen del agua tratada) que igualmente deben ser tratados.

La Spirulina sp es una microalga de fácil manejo a nivel laboratorio, ya que debido a su tamaño sólo requiere un mecanismo de filtrado para su separación, por lo que experimentar con ella resulta accesible para los investigadores.

Se observó que las remociones generadas a partir de biomasa viva y muerta generaron mejores remociones a las 24 horas, lo que podría sugerir que para la biomasa viva se va dando una liberación paulatina de los contaminantes en la medida que la microalga se deteriora o cumple su ciclo de vida. Para la biomasa muerta, ésta libera el cromo a medida que se va degradando, lo cual se lleva a cabo en menor tiempo que en el caso de la biomasa viva.

El que la biomasa retenga el contaminante y luego libere los componentes como parte de un proceso de desorción y/o degradación permite comprender que esta técnica puede ser implementada para el proceso de recuperación de cromo y de este modo evitar que se genere gran cantidad de residuos secundarios por efecto de los tratamientos químicos.

\section{RECOMENDACIONES}

Se recomienda hacer investigaciones con Spirulina $s p$ relacionadas con la remoción de cromo de aguas residuales utilizando el remanente de la biomasa después de la extracción de lípidos para producción de biodiesel, con el fin de incrementar la factibilidad social, económica y ambiental en la producción del biocombustible y el tratamiento de aguas residuales.

Se sugiere hacer un análisis del contenido de cromo en la biomasa de Spirulina $s p$ generada durante las investigaciones enfocadas a la biosorción de este metal en aguas residuales, debido a que resulta importante conocer la cantidad de cromo adsorbido o absorbido por la microalga.

Se aconseja realizar un experimento semejante en tiempos inferiores a las 24 horas, ya que en esta investigación se encontró una 
disminución del porcentaje de remoción al aumentar el tiempo de contacto.

\section{AGRADECIMIENTOS}

Al departamento de Norte de Santander y a Colciencias por brindar su apoyo con la beca de formación de capital humano de alto nivel en el marco de la convocatoria No 753 de 2016.

A Corpocaldas; al CIMAD de la Universidad de Manizales y a la Empresa Relma.

\section{LITERATURA CITADA}

Ardila, L. F. (2012). Medición de la capacidad de Chlorella vulgaris y Scenedesmus acutus para la remoción de cromo de aguas de curtiembre. Obtenido de Universidad Nacional de Colombia: www.bdigital.unal.edu.co/10276/1/299936.2012.pdf

Castañeda, L., \& Castillo, J. (2016). Influencia de la iluminancia, $\mathrm{pH}$ y tiempo en la remoción de sulfuros, Sólidos suspendidos, Demanda quimica y biologica de oxigeno de efluentes de ribera en curtiembres utilizando microalgas en un bioreactor a escala laboratorio. Obtenido de Universidad de Trujillo: http://dspace.unitru.edu.pe

Caviedes, D. I., Muñoz, R. A., Perdomo, A., Rodríguez, D., \& Sandoval, I. J. (2015). Tratamientos para la Remoción de Metales Pesados Comúnmente Presentes en Aguas Residuales Industriales. Una Revisión. Ingenieria y Región, 73-90.

Duque, J. L. (2017). Viabilidad en la producción de biomasa microalgal a partir de fotobioreactores solares en el Valle del Cauca, Colombia. Revista de Investigación Agraria y Ambiental Vol 8, 127-140.

Franco, A. R., Naranjo, J. M., Nieto, D. M., \& Sierra, M. S. (2016). Implementación de un sistema de fitorremediación en zona aledaña a reservaforestal protectora El Malmo, Boyacá, Colombia. Revista de Investigación Agraria y Ambienta, 93-103.

García Hernández, M. d. (Julio de 2014). Remoción de $\mathrm{Cr}(\mathrm{VI})$ de soluciones acuosas por biomasa de Spirulina máxima en un proceso en lote. San $\mathrm{Ni}$ colás de la Garza, Nuevo León, México.

Hernández, A. G., Vázquez-Duhalt, R., Saavedra, M. d., Carreón, L. S., \& Jiménez, A. M. (2009). Biodiesel a Partir de Microalgas. Bio Tecnología, 38-61.
Hong, C., \& Shan-shan, P. (2005). Bioremediation potential of spirulina: toxicity and biosorption studies of lead. Journal of Zhejiang University SCIENCE , 171-174.

Jbari, N. (Septiembre de 2012). Utilización secuencial de microalgas en depuración y adsorción de $\mathrm{Cr}(\mathrm{VI})$. Granada, España: Editorial de la Universidad de Granada.

López, F. A., González, A. R., \& Guzmán, J. M. (2016). Comparación de la reglamentación para el manejo de lodos provenientes de agua residual en Argentina, Chile y Colombia.pdf. Revista de Investigacion Agraria y Ambiental, 227-237.

Martínez, E. R., González, J. C., Juárez, V. M., \& Rodríguez, I. A. (2015). Remoción de Cromo (VI) por una Cepa de Aspergillus niger Resistente a Cromato. Información tecnológica, 13-20.

Martínez, R. I., Paredes, J. F., \& Ordoñes, D. H. (2016). Empleo del estropajo común (Luffa cylindrica) en la remoción de contaminante. Revista de Investigación Agraria y Ambiental, 205-215.

Ministerio de Ambiente y Desarrollo Sostenible (MADS). (17 de Marzo de 2015). Ministerio de Ambiente y Desarrollo Sostenible. Recuperado el 01 de Marzo de 2016, de www.minambiente. gov.co/images/.../app/resoluciones/d1-res_631_ marz_2015.pdf

Organización Mundial de la Salud (OMS). (2006). Guias para la calidad del agua potable primer apendice a la tercera edición. Recomendaciones Organización Mundial de la Salud. Primer apendice a la tercera edición. Volumen 1, 270.

Ortiz, N. E., \& Carmona, J. C. (2015). Aprovechamiento de cromo eliminado en aguas residuales de curtiembres ( San Benito Bogotá) mediante tratamiento con sulfato de sodio. Luna Azul, 117126.

Peña-Hernandez, G. A., Cuesta-Gonzalez, F. A., \& Perez, J. F. (2015). Remoción de carga contaminante en aguas residuales industriales a escala de laboratorio. Revista de Investigación Agraria y Ambiental, 157-168.

Perez, A. H., \& Labbe, J. I. (2014). Microalgas cultivos y beneficios. Revista de Biología Marina y Oceanografia., 157-173.

Tovar, C. t., Ortiz, A. V., \& Jaraba, L. G. (2015). Adsorción de metales pesados en aguas residuales usando materiales de origen biológico. Tecno Lógicas, 109-123. 
